Rev. Elev. Méd. Vét. Poys trop., 1966, 19, 2 (131-136)

\title{
Observation d'une enzootie d'Aspergillose chez des oisons en Haute-Volta
}

\author{
par S. TRAORE ef R. GIDEL
}

\begin{abstract}
RÉSUMÉ
Les auteurs relatent une enzootie d'Aspergillose aviaire en Haute-Volta survenue chez de jeunes oisons importés de France.

Après avoir évoqué dans quelles conditıons ces oiseaux avaient été intraduils, Ils décrivent 'a symplomatologie assez porticulière de l'affection, caractérisée par une prédomınance des manifestations nerveuses. Enfin, après avoir ind:qué les éléments du diagnostic, ils termınent par quelques considérations sur l'étiologie de cette enzootie.
\end{abstract}

\section{I. - INTRODUCTION}

Le service de la production animale en HauteVolta, dont le siège est à Bobo-Dioulasso, $a$, entre autres attributions, celle de promouvoir l'élevage des animaux de basse-cour. Dans ce domaine ses activités consistent à importer notamment des volailles de races et souches amélıorées et à en assurer la diffusion. C'est ainsi que nous avons éfé amenés à envisager l'introduction d'oles dites de Toulouse au Centre avicole de Bobo-Dioulasso.

\section{II. - CONDITIONS D'IMPORTATION ET DE RÉCEPTION DES OISONS AU CENTRE}

Nous nous sommes adressés à notre fournisseur habifuel qui se chargea de l'acquisition, du conditionnement et de l'expédition de 200 oisons (oies de Toulouse, type industriel). En falt les animaux qui nous ont été livrés ne répondaient pas aux critères classiques de ce type, mais se rapprochaient plus du «type agricole».

Deux envois nous ont été adressés dans les conditions suivantes:

L'expédition du premier lot comprenant 70 oisons, réalisée à partir de l'aéroport Paris-Le Bourget, fut effectuée le 16 avril.
Les oiseaux, dont l'arrivée à Bobo-Dioulasso était prévue pour le 17, n'ont pu être effectivement réceptionnés que 24 heures plus tard, par sulte de difficultés techniques rencontrées à Niamey et à Abidjan par l'avion qui les transportalt.

Les anımaux dont nous ignorons les conditions d'entretien au cours des escales, étaient tous en bonne condition au moment de leur réception ef nous n'avons constaté nı fałblesse, ni morbidité, nı mortalité. Ils étalent toutefois très affamés et très assoiffés.

Nntons que les oisons opparemment âgés d'au moins 3 semaınes étoient convenablement conditıonnés dans des boîtes en carton.

Les animaux furent aussitồ placés en poussinière (de $40,80 \mathrm{~m}^{2}$ de surface).

Le deuxième envai a porté sur un lot de 68 sujets. Les animaux expédiés le 23 avrıl, toujours de l'aéroport du Bourget, nous sont parvenus le 24. Leur récepiion eut normalement lieu comme prévu, aucun incident $n$ 'étant intervenu au cours du voyage. Les animaux étolent en bonne santé apparente, toutefois trois d'enire eux présentaient des malformations (cyphose, déformetion de lo hanche avec boiterie, brachygnathie supérieure).

Les sujets manifestement plus jeunes, cor morphologiquement plus petıts que ceux du premier 
lat au moment de leur réception, ne devaient guère avoir plus de 15 jours d'âge.

Le lot fut placé dans une deuxième poussınıère de même superficie que la première et séparée de celie-ci par un hall de 3 mètres de large.

Chaque poussinière comporte un parcours extérieur, les deux parcours étant distants de 4 mètres.

Les lots placés dans les mêmes conditions d'élevage, n'avaient cependant pas de contact direct. Signalons toutefois que le même basse-courier avait à s'occuper des deux poussinières.

Le troisième envoi qui devait immédıatement suivre le second fut annulé, l'enzootie ayant éclaté entre temps.

Notons que l'arrivée des oisons se situe dans la deuxième quinzaine du mois d'avril, un des moments les plus chauds de l'année. C'est également au cours de cette période que l'hygrométrie s'élève progressivement (moyennes des minima et maxima : 21 p. 100 et 61,8 p. $100 ;$ minima et maxima absolus : 5 p. 100 au 15 avril ef 89 p. 100 au 27 avril).

\section{III. _EPIDÉMIOLOGIE_SYMPTOMATOLOGIE}

Le 6 mai au matin, soit environ deux semaines après leur arrivée, nous avons découvert dans la poussinière du $2^{\mathrm{e}}$ lot, le cadavre d'un oison mort au cours de la nuit. Dans la journée du 7, quelques cas de diarrhée étaient observés. Le lendemain, le phénomène diarrhéique devalt s'accentuer avec atteinte d'un plus grand nombre de sujets. Les jaurs suivants, nous devions noter l'apparition de phénomènes nerveux. Cela frappa d'abord un seul sujet, une semaine durant, avant que d'autres ne présentent les mêmes signes.

La première manifestation nerveuse consista en une torsion du cou en $S$ porté soit à gauche, soit à droite, mais toujours d'un seul côté du corps chez le même sujet (photo n⿳1).

Cette torsion du cou se manifestait plusieurs jours duront avant d'être suivie par d'autres symptômes ; parfois même, elle demeurałt le seut signe clinıque. Celo entraînait, chez les sujets atteints, des difficultés d'alimentation et d'abreuvement.

Secondairement, apparaissaient des boiteries et une déformation de la colonne vertébrale suivies de paralysie des pattes. Les animaux, qui ne pouvaient plus se déplacer que par reptation, en se traînant sur le bréchet, gardaient au repos l'attitude du grand écart (photo no 2).

Les manifestations respiratoires sont toujours restées très discrètes. Elles se limitaient à des entrebâillements du bec qui n'avaient lieu que de temps en temps (photo $n^{\circ} 1$ ).

Un total de 13 sujets fut atteint ; 7 succombèrent naturellement ; 6 furent sacrifiés en vue d'effectuer des prélèvements.

Signalons qu'aucune manifestation morbide n'a été observée chez les animaux du premier lot bien que, faute de personnel suffisant, le même basse-courier ait continué à assurer le service des deux poussinières.

\section{IV. - DIAGNOSTIC NÉCROPSIQUE}

Tous les animaux morts ou sacrifiés furent l'objet d'une autopsie systématıque. Les lésions macroscopiques constatées étaient limitées et ne présentaient que peu de rapports avec les mani festations cliniques observées. Elles se situaient en effet essentiellement au niveau des poumons avec atteinte soit d'un seul, soit des deux organes à la fois. La trachée par contre présentait un aspect normal. Aucune lésion macroscopique ne pût être décelée au niveau du cerveau et de la moelle épinière.

Les lésions pulmonaires se présentaient sous forme de petites granulations en amas, de couleur rouge-vin et de la taille d'une tête d'épingle. Ces granulations se rencontraient plus particulièrement sur la face costale du poumon atteint.Cependant, on n'observait ni adhérence, ni hépatisation. Les photos effectlées le furent en noir et blane et sont, de ce fait, d'une interprétation difficile. Nous avons donc renoncé à en faire état dans cette publication.

En présence de ces lésions, nous avons été amenés à évoquer le diagnostic de suspicion d'Aspergillose.

\section{V. - DIAGNOSTIC DE LABORATOIRE}

Le tissu pulmonaire des régions lésées fut prélevé et, étant donné l'existence des manifestations nerveuses, on procéda également à des prélèvements de moelle cervicale. Toutes ces opérations furent effectuées de façon stérile. 
Au laboratoire, les prélèvements firent d'abord l'objet d'un examen microscopique direct qui permit de mettre en évidence la présence de spores et de quelques filaments mycéliens dans les lésions pulmonaires. Des ensemencements du tissu pulmonaire et de la moelle furent ensuite effectués après broyage sur gélose Sabouraud glucosée + actidione, gélose Sabouraud maltosée et gélose Sabouraud dextrosée.

Des colonies vertes et veloutées, avec touffes de filaments aériens cotonneux blancs, apparurent en 24 heures dans tous les milieux ensemencés. Ces colonies examinées au microscope montrèrent qu'elles étaient constituées de nombreux filaments mycéliens, de spores libres et de têtes aspergillaires ayant la morphologie d'aspergil.us fumigatus (photo no 3 ). Un lapin inoculé par vole intraveineuse avec une suspension de spores mourut quatre jours plus tard.

Ces résultats nous incitèrent à confirmer le diagnostic d'aspergillose.

\section{VI. - MESURES PROPHYLACTIQUES}

Le traitement de l'aspergillose étant reconnu comme illusoire, aucun fongicide n'ayant à I'heure actuelle une action efficace contre Aspergillus fumigatus, nous n'avons mis en œuvre que des mesures prophylactiques.

Dans un premier temps, il fut procédé à l'isolement de tous les malades et suspects. Ainsi, nous pouvions suivre les manifestations et l'évolution de la maladie. Les sujets morts firent l'objet d'une autopsie systématique.

Ultérieurement tous les malades et suspects survivonts furent sacrifiés en vue des prélèvements.

Enfin des doses fortes de vitamines (Dodecavit) furent admınistrées au reste du troupeau.

Ces dispositions permirent à notre avis, de limiter l'extension de la maladie, car aucune manifestation morbide ne fut constatée chez les autres sujets.

\section{VII. - ETIOLOGIE}

Si l'agent causal a pu être identifié, nous en sommes réduits à des hypothèses quant aux conditions d'introduction de cet agent dans notre élevage. Aucun cas d'aspergillose n'a été observé dans nos stations depuis leur création en 1953, ni signalé par les agents du Service au niveau des élevages particuliers de la région de BoboDioulasso, bien que des oiseaux de la même race aient été importés et diffusés au cours des années antérieures.

Ultérieurement et après lavage et désinfection à la soude caustique, à la lampe à souder et au titanol (") sulvis d'un badigeonnage des murs à la chaux vive, un lot de Leghorn et de RhodeIsland a été installé dans le même local. A quatre mois d'age, ces oiseaux n'ont présenté aucune manifestation d'aspergillose.

Nous sommes donc amenés à penser que les oisons, ou du moins certains d'entre eux, étalent porteurs de germes au moment de leur introduction dans notre élevage. Cela est d'autant plus orncevable que nous savons qu'il existe un cycle d'infection allant de l'adulte à l'embryon en passant par l'intermédiaire de l'œuf à couver.

Par ailleurs l'existence des malformations signalées plus haut nous incitent à penser que les animaux du second lot provenaient d'une bande plus fragile et plus prédisposée.

D'autre part les conditions climatiques évoquées précédemment ont pu jouer un rôle favorisant dans l'étiologie de cette enzootie.

\section{VIII. - CONCLUSION}

11 nous. a paru intéressant de rapporter cette observation, car c'est la première fois que l'aspergillose aviaire est signalée en Haute-Volta.

Nous retiendrons l'allure particulière de cette enzootie, avec ses manifestations nerveuses prédominantes et l'absence presque' complète de signes respiratoires en opposition avec les lésions constatées.

Quant à l'étıologre, elle reste dons le domaine des hypothèses.

\section{Service de lo production Animale. Centre Muraz - O.C.C.G.E. Bobo-Dioulasso (Haute-Volta).}

* Laboratoires King d̀ Marseille. 


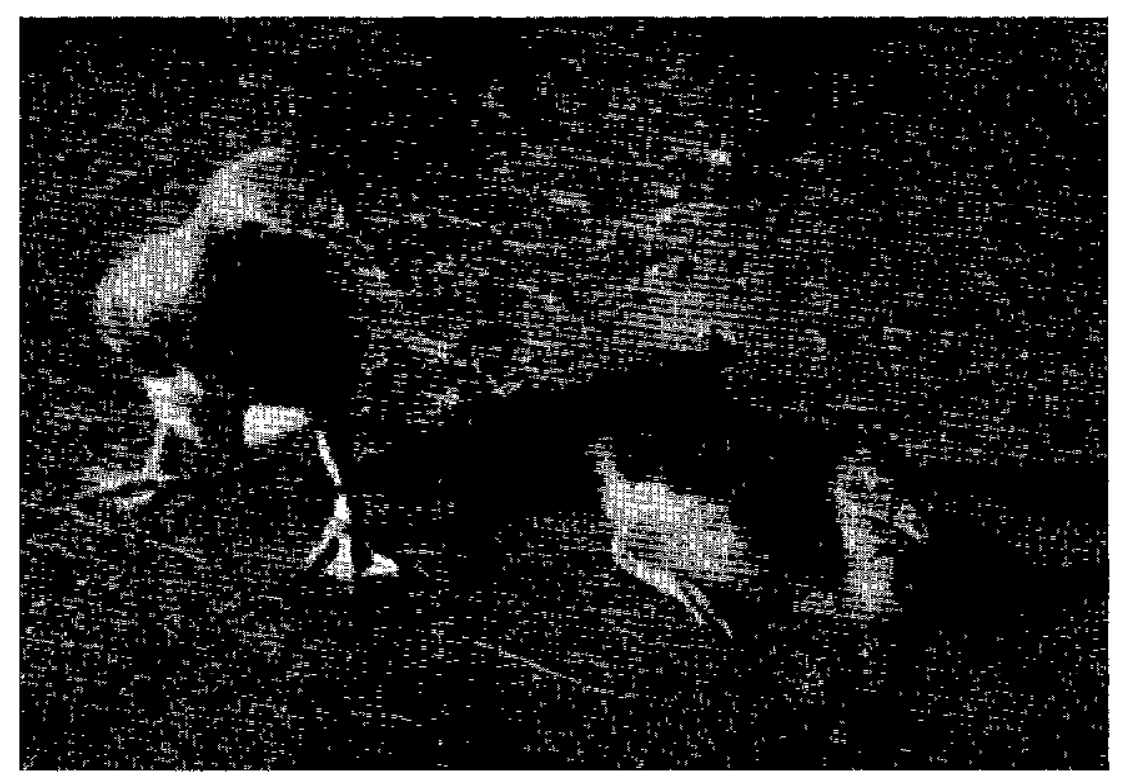

Cliché no 1. — \&ison présentant une torsion du cou et entrebôillement du bec ».

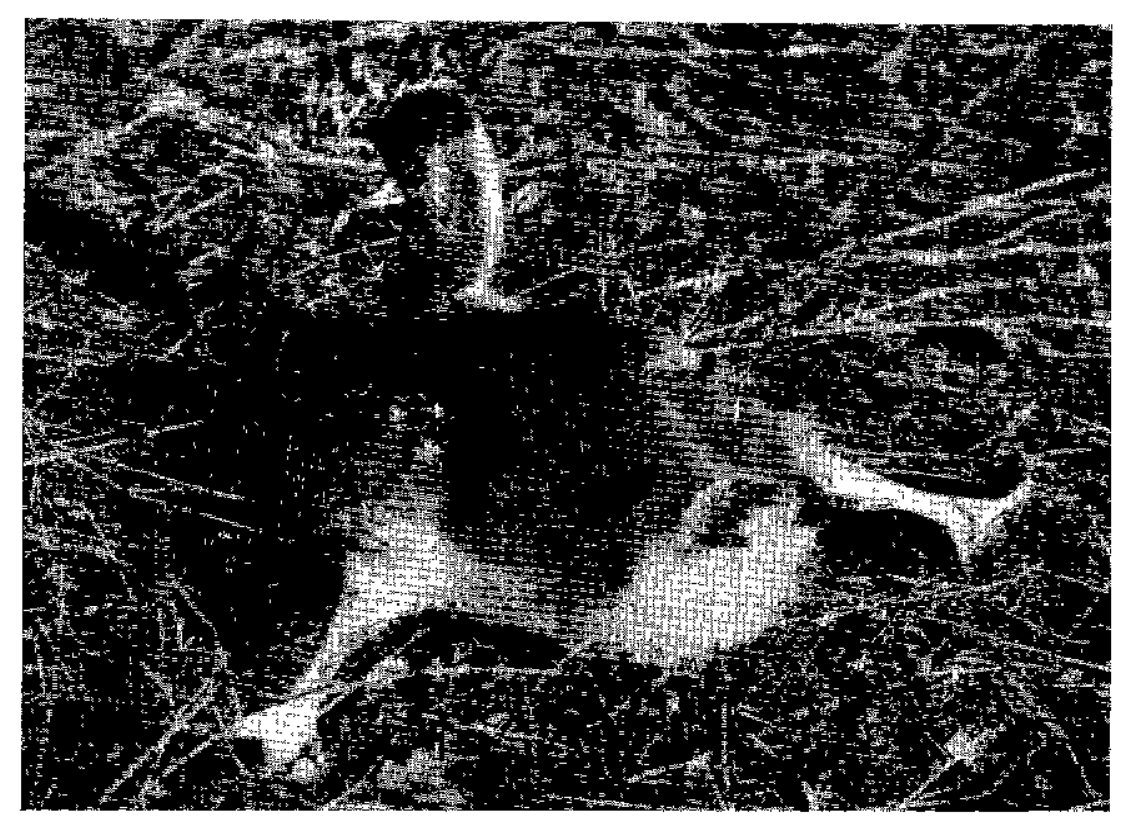

Cliché $n^{0} 2 .-$ « Oison présentant une paralysie des partes avec attılude du grand écart $»$. 


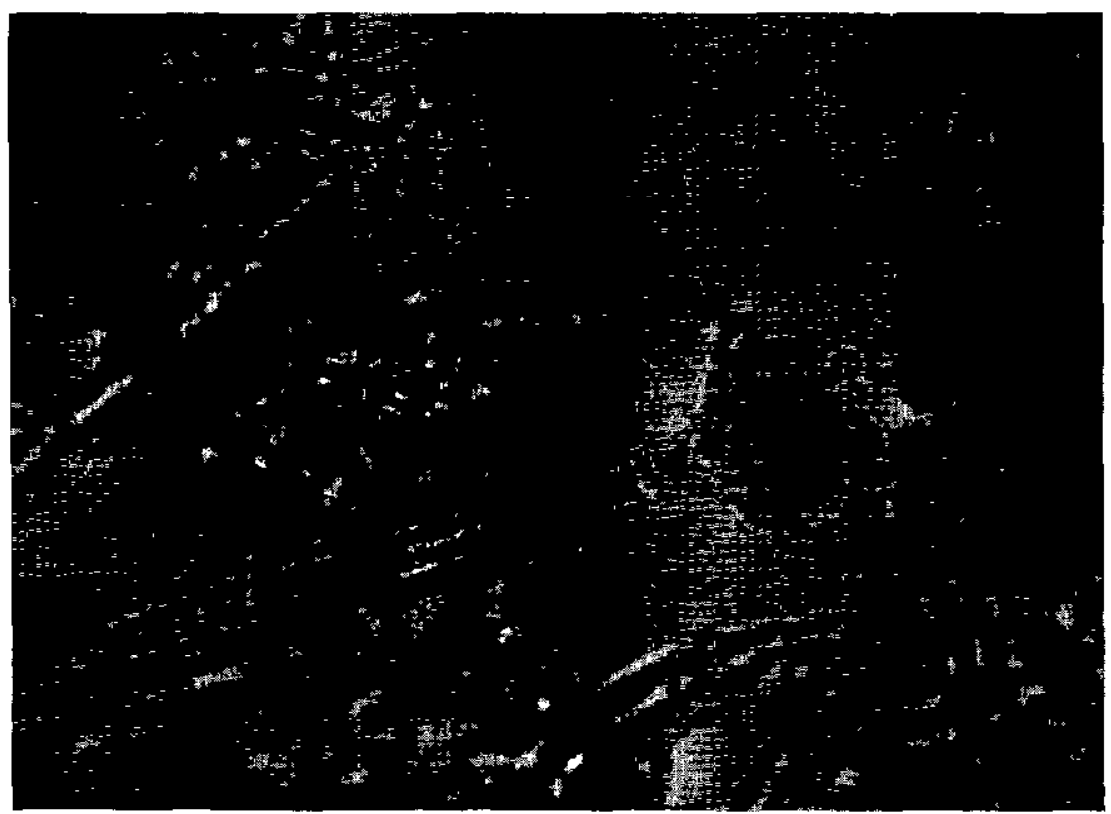

Cliché n 3. - « Spores, filaments mycéliens et têtes aspergillaires ».

\section{SUMMARY}

\section{Observation on an outbreak of avian Aspergillosis in Upper Volta}

An outbreak of avian Aspergillosis was reported in Upper Volta in young goslings imported from France. The circumstances in which these birds were imported are mentionned and the rather peculiar symptomatology of this infection, which is marked by the prevalence of the nervous signs, is described. Then, some data are given for the diagnosis and the etiology of this disease is pointed out.

\section{RESUMEN}

Observación sobre una enzootia de aspergilosis en los gansos pequeños en Alta-Volta

Los autores notan en Alta-Valta, una epızootic de aspergilosis aviar encontrada en gansos pequeños imporiados de Francia.

Evocan las condiciones de introducción de esias aves $y$ describen la sıntomatología muy particular de esta enfermedad, caracterizoda por un predaninio de las manifestaciones nerviosas. Luego, indican los elementos del diagnóstico y concluyen a aigunas considernciones sobre la eliologia de esia enzootia. 


\section{BIBLIOGRAPHIE}

DROUHET (E.). - Les actualités thérapeutiques des mycoses chez l'Homme. Rev. Poth. Comp. 1965, 2, n० 3, 177-186.

EUZEBY (J.). - Le Parasitisme en pathologie aviaire. Paris Vigot 1960.

FRITZSCHE (B.) et GERRIETS (E.). - Maladies des volailles, Paris, Vigot, 1965.

GUILHON (J.) et JOLIVET (G.). - Thérapeutique des mycoses animales. Rev. Path. Comp. 1965, 2, no 3, 187-193.

LESBOUYRIES (G.). - La pathologie des oiseaux. Paris, Vigot, 1941.
OLIVIER (H. R.). - Traité de Biologie Appliquée. Tome II. Paris, Maloine, 1963.

REIVAULT (L.), GUILLOON (J. C.), MAIRE (C.). Candidose de la pintade. A propos de quelques observations. Rev. Med. Vet. 1965, 141. n० 4, 363-366.

SAEZ (H.). - Aspergillus fumigofus Fresenius isolé chez l'animal. Analyse portant sur quatre années de recherches. Ann. Parasit. Hum. Comp. 1965, 40, 1, 105-118.

SEGRETAIN (G.), DROUHET (E.), MARIAT (F.). - Diagnostic de Laboratoire en mycologie médicale. Porıs, Edition de la Tourelle, 1964. 